\title{
Plasmaspheric electron density estimation based on COSMIC/FORMOSAT-3 data
}

Fabricio Prol ${ }^{1}$, Mainul Hoque ${ }^{1}$

${ }^{1}$ German Aerospace Center (DLR), Germany Institut für Solar-Terrestrische Physik (SO-WWB)

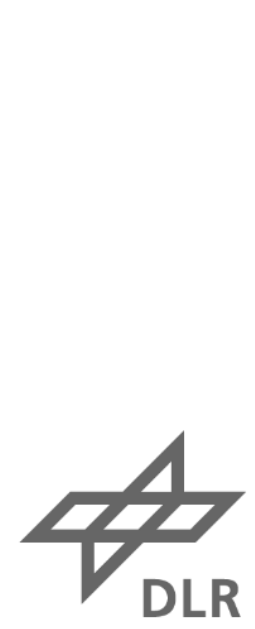




\section{Summary}

1. Method Explanation

2. Results of the Method

3. Conclusions

4. Future 


\section{Method}

We have used the most traditional tomography method, but with some adaptations.

$$
\begin{gathered}
\text { ART } \\
x_{j}^{k+1}=x_{j}^{k}+\gamma \frac{\left(\mathrm{y}_{\mathrm{i}}-\sum_{j}^{N} A_{i j} x_{j}^{k}\right)}{\sum_{j}^{N} A_{i j}^{2}} A_{i j} \\
\gamma_{i j}=0.2\left(x_{j}^{0} / x_{\text {max }}^{0}\right)^{w_{\phi_{m}}}
\end{gathered}
$$

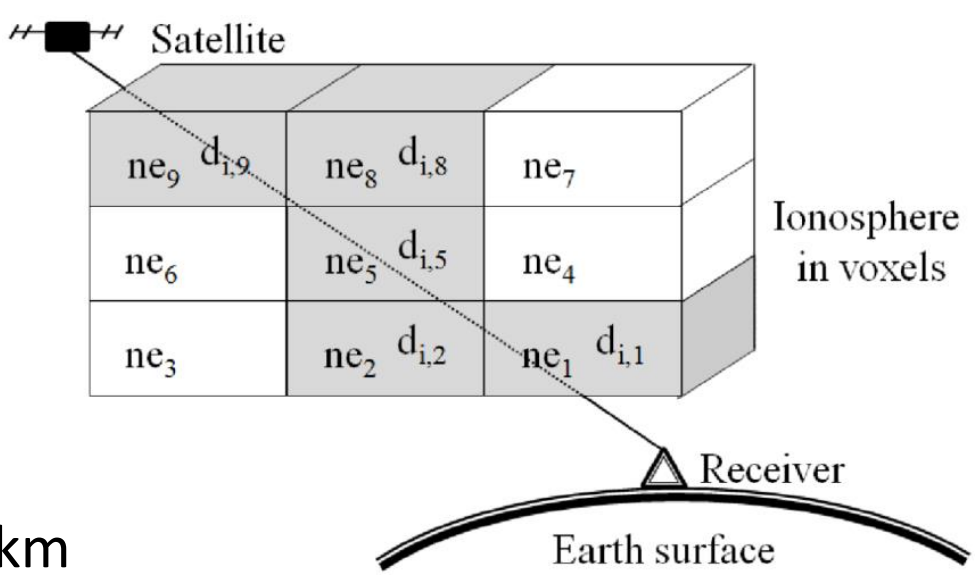

Resolution: daily maps with $2^{\circ} \times 15^{\circ} \times 50 \mathrm{~km}$

Background: given by NPSM${ }^{1}$ (in-house plasmasphere model).

1. N. Jakowski, and M. M. Hoque, "A new electron density model of the plasmasphere for operational applications and services," J. Space Weather Space Clim., vol. 8, pp. A16, 2018. 


\section{Method}

Indeed, some regularization is needed. Otherwise, one can observe artifacts. The examples are shown using IRI, NeQuick and NPSM as background.
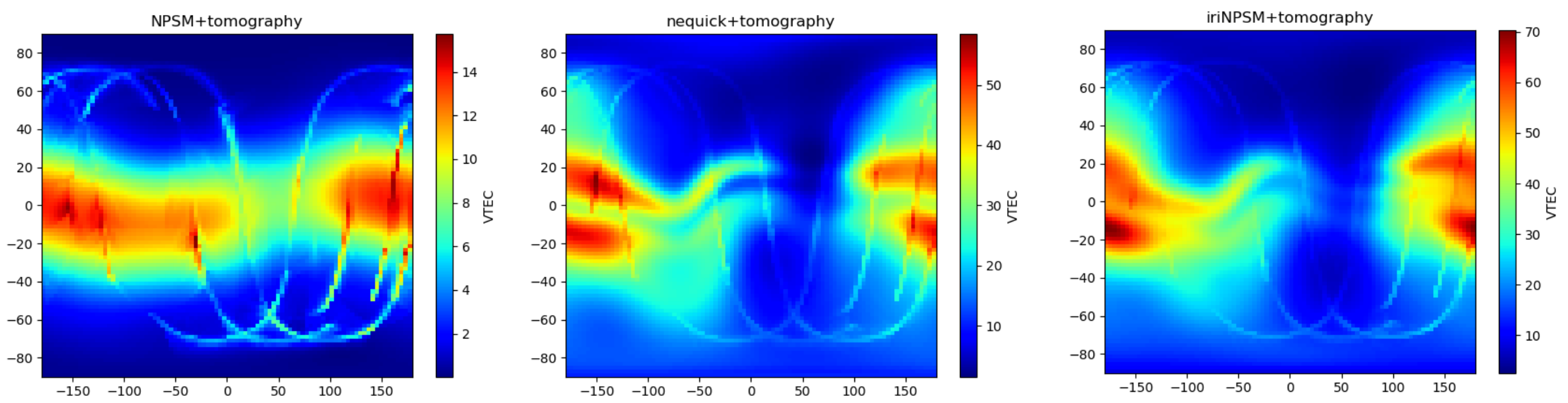

Fig. 1 - VTEC maps using TEC from POD* data as input to the tomography.

*POD stands for Precise Orbit Determination

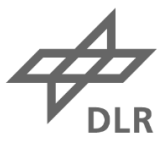




\section{Method}

Artifacts also remains in the meridional sections of the plasmasphere
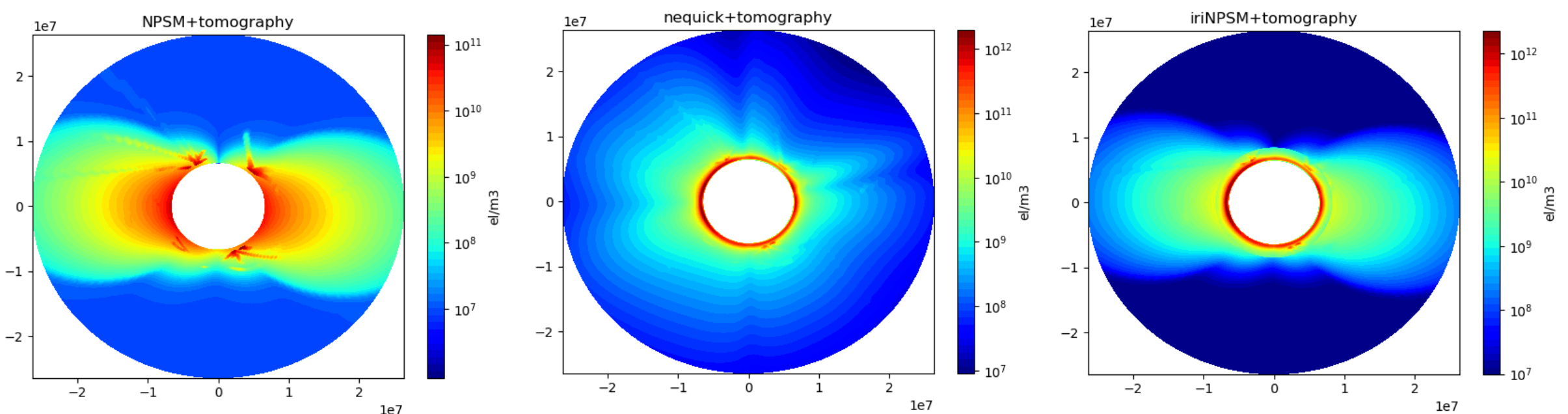

Fig. 2 - Electron density slices using TEC from POD data as input to the tomography.

Regularization: similar to the one provided by Heise et al 2002.

2. Heise S, Jakowski N, Wehrenpfennig A, Reigber C, H Lühr (2002) Sounding of the topside ionosphere/plasmasphere based on GPS measurements from CHAMP: Initial results. Geophys. Res. Lett., 29:1699.

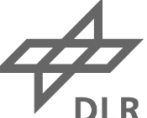




\section{Results}

After regularization we can observe a much more smoothed ionosphere (click in the space bellow to run the video).

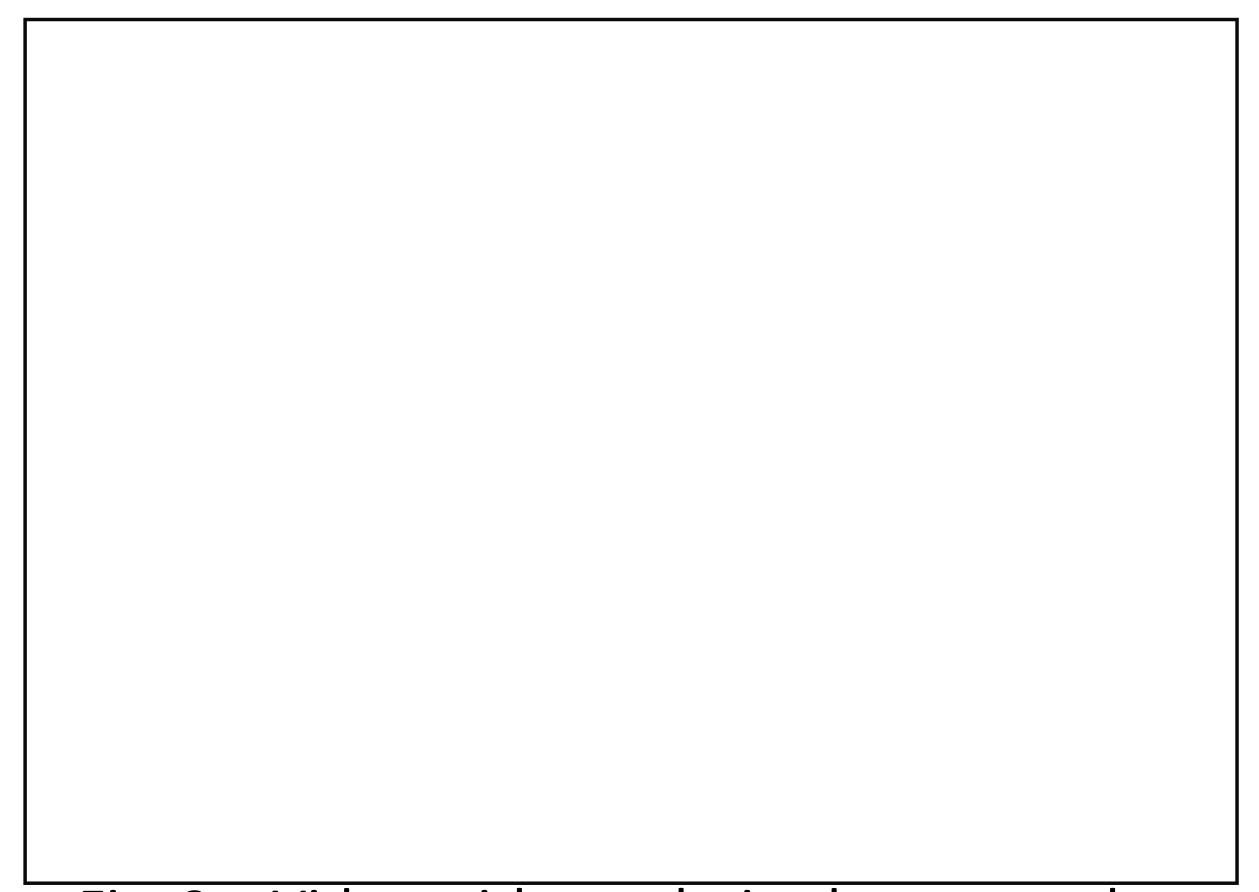

Fig. 3 - Video with regularized tomography.

video link: https://drive.google.com/file/d/1KZXet8HAfIFXJKA-p609 TD-F9-P2DGT/view 


\section{Results}

An comparison with DMSP data is given by the Fig. bellow.

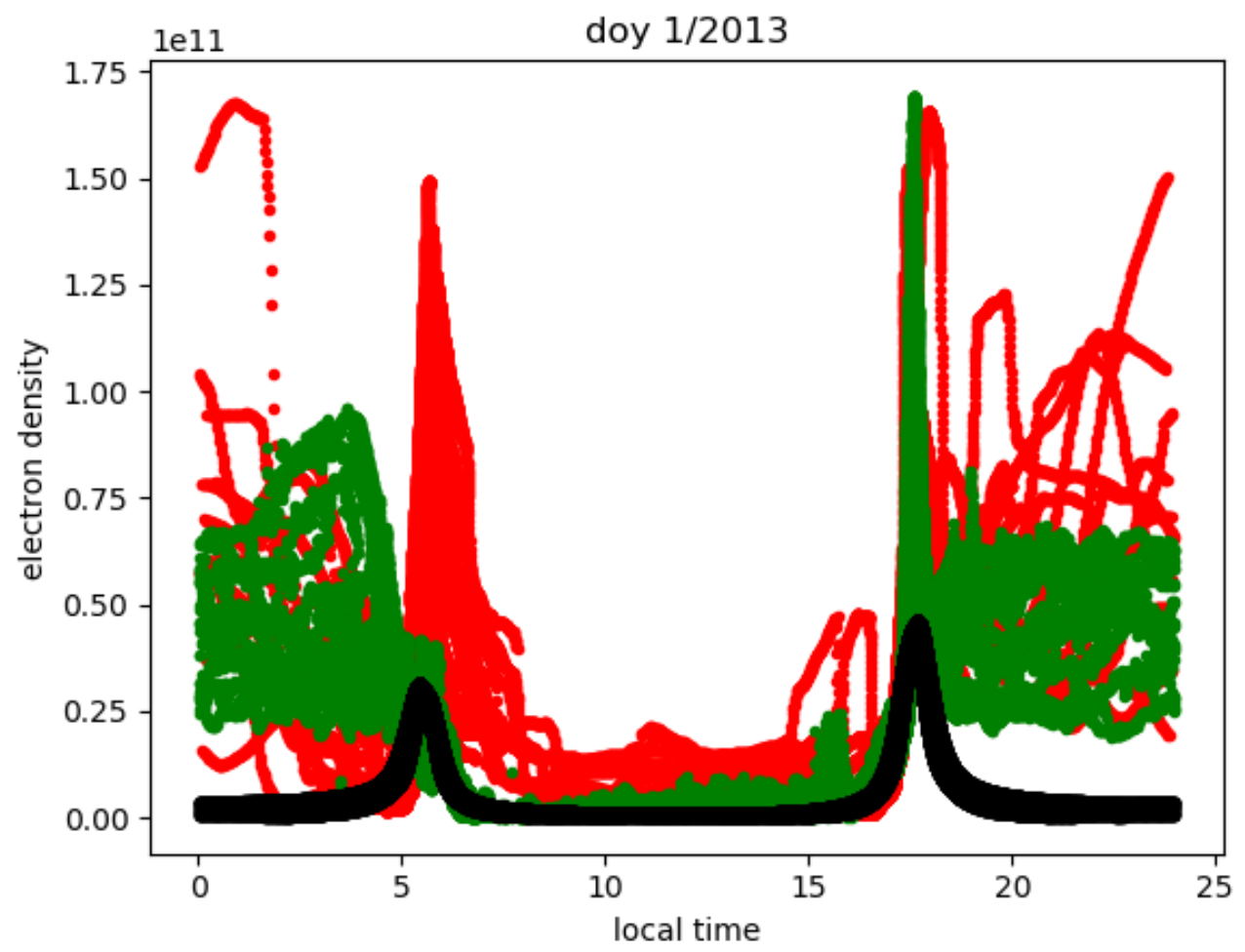

red: DMSP

green: tomography black: background

Fig. 4 - Tomography vs DMSP (Defense Meteorological Satellite Program).

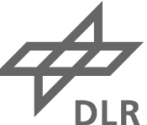




\section{Results}

An assessment of several days was conducted during the entire years of 2008 and 2013. The table bellow show some general results.

Tab. 1 - Tomography vs DMSP (2008).

\begin{tabular}{ccc}
\hline & Tomography & Background \\
\hline Mean Error & 0.62 & 0.52 \\
Std & 0.82 & 1.22 \\
\hline
\end{tabular}

units: $10^{10} \mathrm{el} / \mathrm{m}^{3}$
Tab. 1 - Tomography vs DMSP (2013).

Tomography Background

\begin{tabular}{ccc}
\hline Mean Error & 0.25 & -0.4 \\
Std & 2.05 & 2.56 \\
\hline
\end{tabular}

units: $10^{10} \mathrm{el} / \mathrm{m}^{3}$

Tomography has provided an improvement of $20 \%$

in comparison to the background. 


\section{Conclusions}

- It is possible to apply plasmasphere tomography based on a single satellite from COSMIC

- Natural variability of the ambient plasma was well represented in terms of latitude, altitude, solar activity, and local time.

- Poor-quality estimations occurred in nighttime at high-latitude regions due to the ill-conditioned geometry, poor specification of the background and measurement errors in COSMIC TEC in the order of a few TECU.

- General Root Mean Square Ratio (RMSE) improvement higher than $20 \%$ was obtained in TEC and electron density in comparison to the background. 


\section{Future}

Next steps: incorporation of new constellations as well as RO$\mathrm{Ne}$ into the background model.

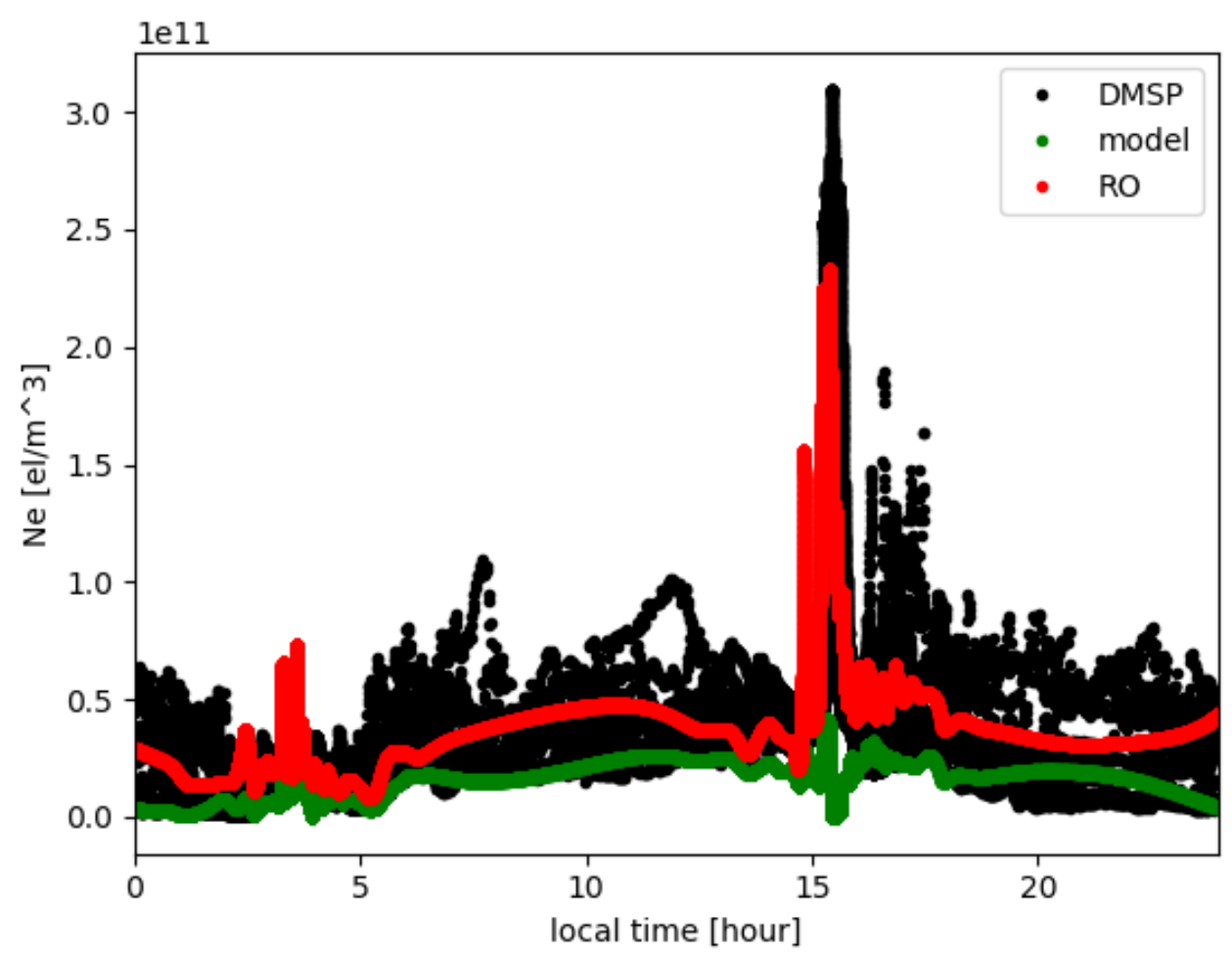




\section{Thank You!}

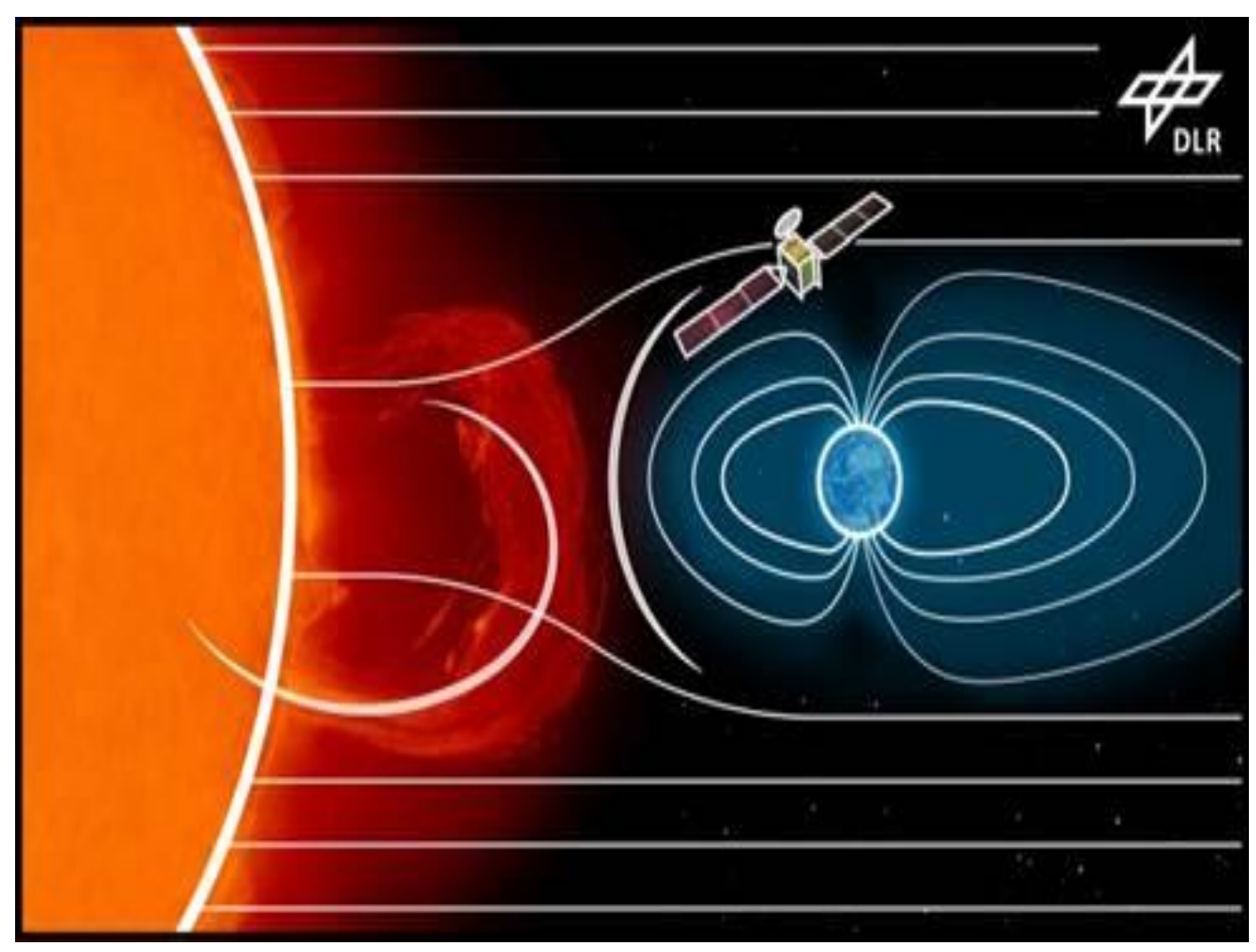

$\oiint_{\text {DLR }}$ 